\title{
Análise retrospectiva de 20 pacientes acometidos por schwannoma nos membros superior e inferior*
}

\section{Retrospective Analysis of 20 Patients Affected by Schwannomas in the Upper and Lower Limbs}

\author{
José Antonio Galbiatti ${ }^{10}$ Gabriel Rodrigues dos Santos Milhomens ${ }^{1}{ }^{\circledR} \quad$ Lara Giovanetti Bertozzo ${ }^{10}$

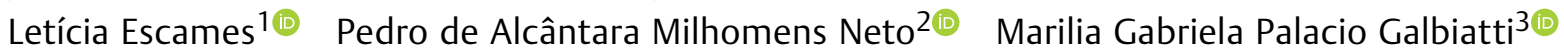

${ }^{1}$ Faculdade de Medicina de Marília, Marília, SP, Brasil
2 Faculdade de Medicina de Jundiaí, Jundiaí, SP, Brasil
${ }^{3}$ Faculdade de Medicina, Universidade de Marília, Marília, SP, Brasil

Rev Bras Ortop 2020;55(5):629-636.
Endereço para correspondência Gabriel Rodrigues dos Santos Milhomens, Faculdade de Medicina de Marília, Rua Hidekichi Nomura, 125, apto. 34, Fragata, Marília, SP, 17519-221, Brasil (e-mail: gmilhomens5@hotmail.com).

\section{Resumo}

Palavras-chave

- neoplasias

- nervos periféricos

- neurilemoma
Objetivo Analisar o perfil epidemiológico e a evolução de 20 pacientes diagnosticados com schwannoma nos membros superiores e inferiores.

Métodos Definiu-se um grupo de pacientes para avaliação retrospectiva, compreendendo o período entre fevereiro de 2002 e junho de 2018, no qual foram estudados e avaliados 20 prontuários de pacientes submetidos a procedimento cirúrgico devido a schwannoma; a confirmação diagnóstica foi feita pelo exame anatomopatológico.

Resultados Tanto os pacientes do sexo masculino quanto do feminino foram igualmente acometidos, e a média de idade foi de 50,85 anos, variando de 12 a 77 anos. Houve predomínio do membro superior e da face flexora. O nervo mais acometido foi o ulnar, e 6 (30\%) pacientes apresentaram complicações pós-operatórias transitórias. Não foi identificado nenhum caso de recidiva tumoral.

Conclusão O schwannoma é uma lesão rara e de difícil diagnóstico. Deve sempre ser considerada como hipótese quando se estiver diante de um tumor de partes moles acometendo os membros. O sinal de Tinel deve ser levado em consideração por conta de sua maior correlação com as complicações. Os pacientes devem ser informados quanto às possíveis complicações pós-operatórias, que são frequentes, mas, geralmente, transitórias.

Trabalho desenvolvido no Departamento de Ortopedia e Traumatologia, Irmandade da Santa Casa de Misericórdia de Marília, Marília, SP, Brasil.

recebido

09 de Junho de 2019

aceito

05 de Dezembro de 2019
DOI https://doi.org/

10.1055/s-0040-1702962. ISSN 0102-3616.
Copyright $\odot 2020$ by Sociedade Brasileira License terms de Ortopedia e Traumatologia. Published by Thieme Revinter Publicações Ltda, Rio de Janeiro, Brazil 


\section{Keywords \\ - neoplasia \\ - peripheral nerves \\ - neurilemmoma}

20 medical records of patients undergoing surgery due to schwannoma; the diagnosis was confirmed by an anatomopathological examination.

Results Male and female patients were equally affected. The average age was 50.85 years, ranging from 12 to 77 years. There was a predominance of the upper limb and of the flexor face. The most affected nerve was the ulnar nerve. In total, 6 (30\%) patients had transient postoperative complications. No cases of tumor recurrence were identified.

Conclusion Schwannoma is a rare and difficult-to-diagnose lesion. It should always be considered as a hypothesis when facing a soft-tissue tumor affecting the limbs. The Tinel sign should be regarded, given its higher correlation with complications. The patients should be informed of the possible postoperative complications, which are frequent but usually transient.

\section{Introdução}

O schwannoma, também denominado neurilemoma, foi descrito primeiramente por Verocay em $1908 .^{1}$ Origina-se das células de Schwann e, embora raro, é o tumor benigno mais comum da bainha dos nervos periféricos, compreendendo $5 \%$ de todos os tumores de partes moles. Geralmente apresenta-se como um tumor solitário, apesar de já terem sido descritos na literatura casos de múltiplos tumores. É mais comum entre 30 e 60 anos, sem predileção para sexo ou raça. Ocorrem mais comumente na cabeça e no pescoço, seguido do tronco, e posteriormente dos membros superiores e inferiores. $^{2-8}$ O membro superior é acometido em até $19 \%$ dos casos, enquanto o inferior, em até $17,5 \% .{ }^{1}$ Há predominância da face flexora, devido à maior concentração de fibras nervosas nessa região, com preferência para os nervos mistos. ${ }^{6,8,9}$ Os nervos mais acometidos são o ulnar e o mediano, no membro superior, e o fibular e o tibial posterior, no membro inferior. ${ }^{1,10-12}$

Embora a etiologia ainda não esteja totalmente esclarecida, há certa evidência para anormalidades cromossômicas como a principal causa, em especial no cromossomo $22 .{ }^{13,14}$

O schwannoma apresenta-se clinicamente como uma massa oval de consistência firme, excêntrica ao nervo, com diâmetro geralmente menor do que $3 \mathrm{~cm}$, bem encapsulada, com crescimento lento e padrão não infiltrativo, de superfície lisa e coloração amarelo-acinzentada ou cinza-esbranquiçada. Pode persistir como um edema indolor por anos, antes de surgirem outros sintomas, tais como dor, parestesia, hipoestesia e déficit motor, que são decorrentes da compressão de estruturas adjacentes e da ausência da distensibilidade tecidual local. $3,8,13,15,16$

À microscopia, podem ser distinguidos dois tipos de tecido: Antoni-A e Antoni-B. O primeiro caracteriza-se por células fusiformes com limites indistintos e núcleos deformados ou ondulados, dispostas em folhas ou feixes, e com mitoses esparsas. Há arranjos em paliçada e áreas anucleadas entre eles, o que caracteriza os corpos de Verocay. Já o segundo é menos celular e sem características arquitetônicas distintas; sua matriz contém colágeno esparso, numerosos vasos sanguíneos, e uma mistura de células fusiformes e ovais. Focos de células inflamatórias, incluindo histiócitos, podem estar pre- sentes, e alguns contêm hemossiderina. Usualmente, há predomínio de um tipo histológico em cada lesão. É comum a presença de degenerações decorrentes da hialinização da parede dos vasos, hemorragia e deposição de fibrina, com consequente formação de cistos, fibrose da matriz e calcificação focal. O núcleo das células de Schwann torna-se hipercromático e multilobulado, e o tumor apresenta-se com uma coloração amarelo-acinzentada nesses casos. ${ }^{1,3,16-18}$

Ao exame físico, a massa é dolorosa à pressão e móvel no eixo transversal, mas não no eixo longitudinal do membro; a percussão do tumor induz parestesia dolorosa na área do nervo, similar a um sinal de Tinel positivo.,15

Entre os exames complementares, a ultrassonografia e a ressonância magnética podem ser utilizadas para auxiliar no diagnóstico. Esta última é o exame de imagem de preferência. Pode-se ainda utilizar testes imunohistoquímicos, que evidenciam imunopositividade para as proteínas S-100 e Leu-7. No entanto, a confirmação diagnóstica só é realizada por meio do exame anatomopatológico. 1,14,16,17,19

Como diagnósticos diferenciais, podemos citar gânglio, tenossinovite, neurofibroma, lipoma, xantoma, mixoma, hemangioma, e tumor de células gigantes da bainha do tendão. $4,7,19,20$

O tratamento de escolha é a excisão cirúrgica precoce por meio da técnica intra ou extracapsular e tem como objetivo remover o tumor e preservar a função do nervo. A cirurgia é indicada nos casos sintomáticos e ainda não há um consenso sobre qual é a melhor técnica. ${ }^{1,3,6,7}$ Embora tenha um bom prognóstico, distúrbios sensitivos e/ou motores temporários no pós-operatório, tais como hipoestesia e parestesia, são vastamente relatados na literatura. Esses distúrbios são decorrentes da dissecção ou retração do nervo, e não interferem significativamente nas atividades diárias. 2,6,19

O objetivo deste trabalho é analisar o perfil epidemiológico e a evolução de 20 pacientes diagnosticados com schwannoma entre 2002 e 2018.

\section{Materiais e Métodos}

Definiu-se um grupo de pacientes para avaliação retrospectiva, compreendendo o período entre fevereiro de 2002 e 

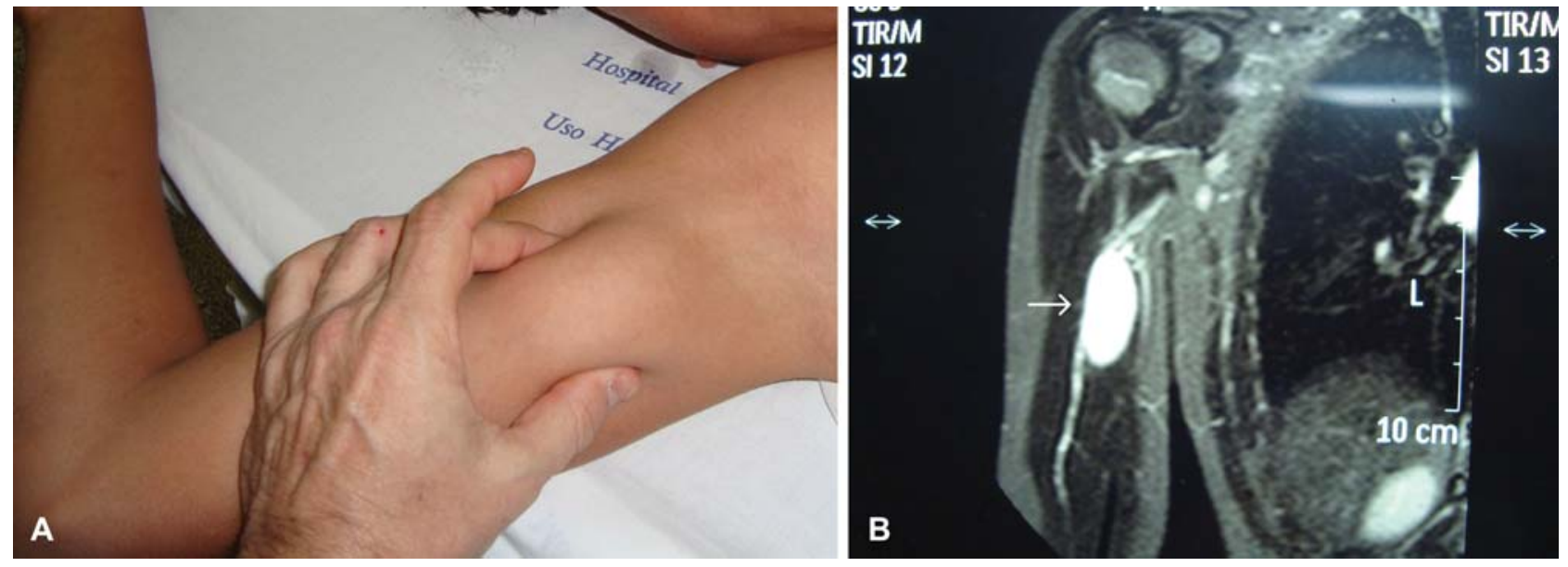

Fig. 1 Fotografias pré-operatórias do paciente número 5, acometido por schwannoma no braço direito. (A) Vista superior de braço direito com tumoração em região medial destacada por palpação. (B) Fotografia de ressonância magnética de membro superior direito, com seta indicando schwannoma em nervo cutâneo medial do braço.

junho de 2018, no qual foram estudados e avaliados 20 prontuários de pacientes submetidos a procedimento cirúrgico devido a schwannoma.

Os critérios de inclusão foram: cirurgias de tumores dos membros superiores e inferiores, realizadas no Serviço de Ortopedia da nossa instituição no período pré-definido, que apresentassem confirmação diagnóstica de schwannoma pelo exame anatomopatológico.

Foi atribuído um número de ordem cronológica aos pacientes a partir da data do tratamento cirúrgico.

Para auxiliar na elucidação diagnóstica, foi realizada ressonância magnética e/ou ultrassonografia. Posteriormente, a hipótese diagnóstica foi confirmada pelo resultado do exame anatomopatológico.

O tratamento de escolha foi a excisão completa do tumor. Todos os procedimentos foram realizados com técnica microcirúrgica, isto é, foram utilizados equipamentos para ampliação do campo cirúrgico, como lupas com magnificação de pelo menos quatro vezes e/ou microscópio cirúrgico. Além disso, os instrumentos utilizados eram delicados e específicos para cirurgia dos nervos periféricos.

A partir das informações oferecidas pelos exames de ressonância magnética e/ou ultrassonografia, a via de acesso foi sempre planejada considerando a região do tumor, centrando-a sobre o mesmo (- Figura 1). Quando atingimos os planos mais profundos, sempre iniciamos a dissecção do nervo nas regiões proximal e distal ao tumor, onde o nervo está "normal." A seguir, isolamos o nervo com o auxílio de drenos de Penrose finos (-Figuras 2, 3 e 4), e iniciamos a ressecção efetiva do tumor, por meio da técnica intra ou extracapsular. Pode-se usar o plano de clivagem entre as fibras neurais que não foram afetadas pelo schwannoma. Neste tempo cirúrgico, muitas vezes é necessário avaliar o nervo de forma circunferencial durante a exérese do tumor. A cirurgia tem como objetivo remover o tumor por completo e preservar a função do nervo.

Os pacientes foram avaliados a partir de um protocolo previamente elaborado pelos autores. A partir dos dados dos
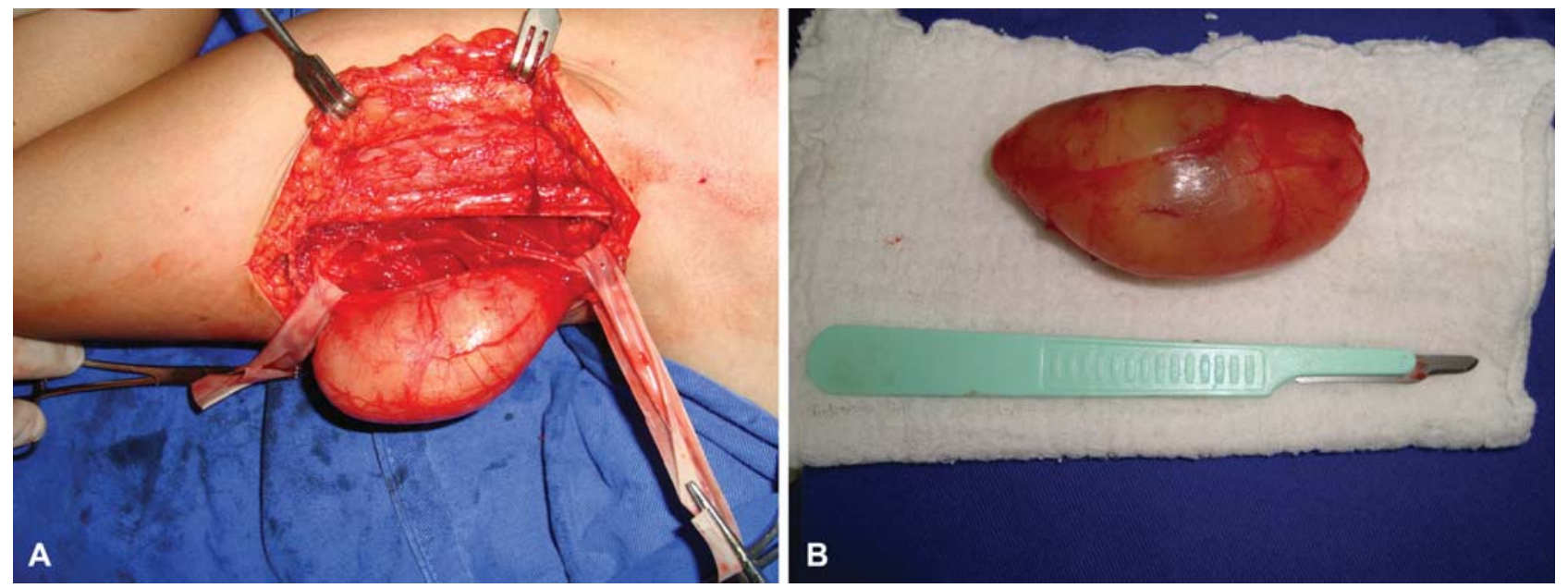

Fig. 2 Fotografias perioperatórias durante e após dissecção de tumor em região medial de braço direito (paciente número 5). (A) Nota-se a utilização de drenos de Penrose para isolamento do nervo, permitindo manter a integridade das estruturas vásculo-nervosas durante o ato cirúrgico. (B) Peça tumoral após exérese, apresentando-se como uma grande massa ovalada, de coloração amarelo-acinzentada. Ao lado, bisturi para comparação de dimensões. 

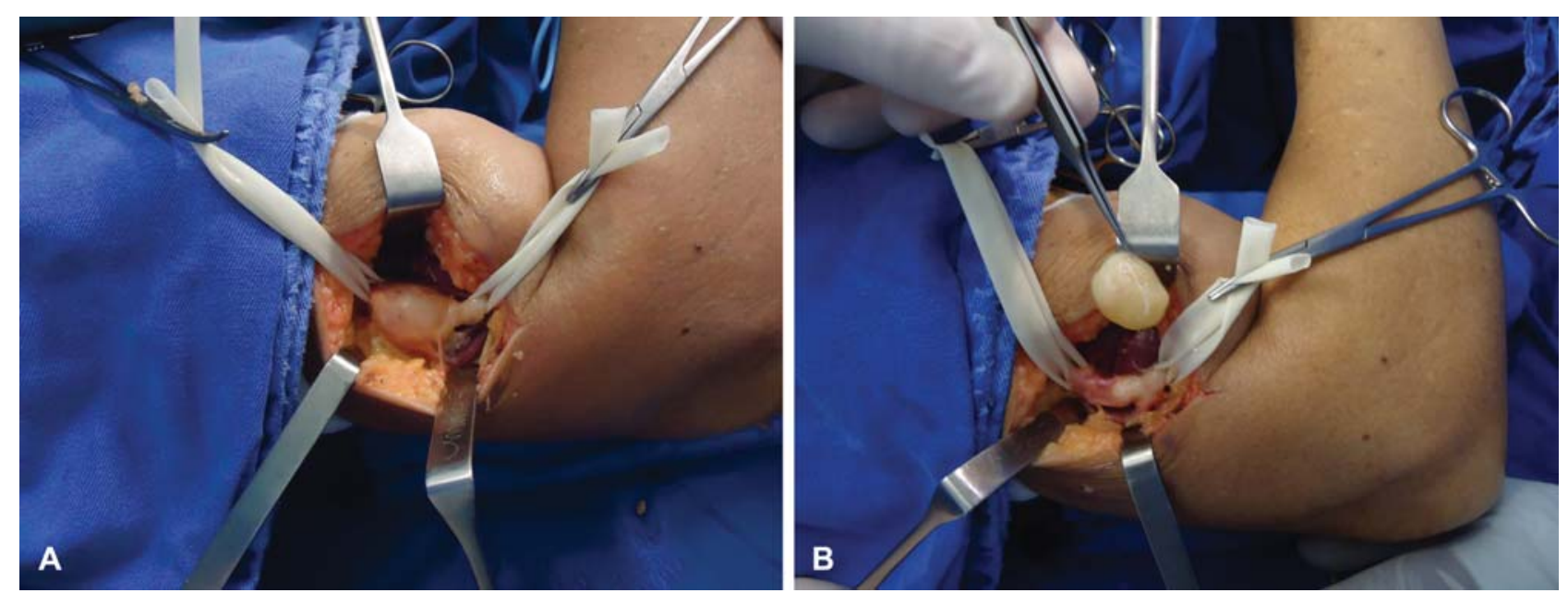

Fig. 3 Fotografias intraoperatórias de vista lateral de retirada de schwannoma em braço direito (paciente número 9). (A) Pode-se observar a utilização de drenos de Penrose para isolamento do nervo radial, proximal e distalmente ao tumor. (B) Reparo do nervo, notando-se externamente o tumor retirado em sua totalidade.
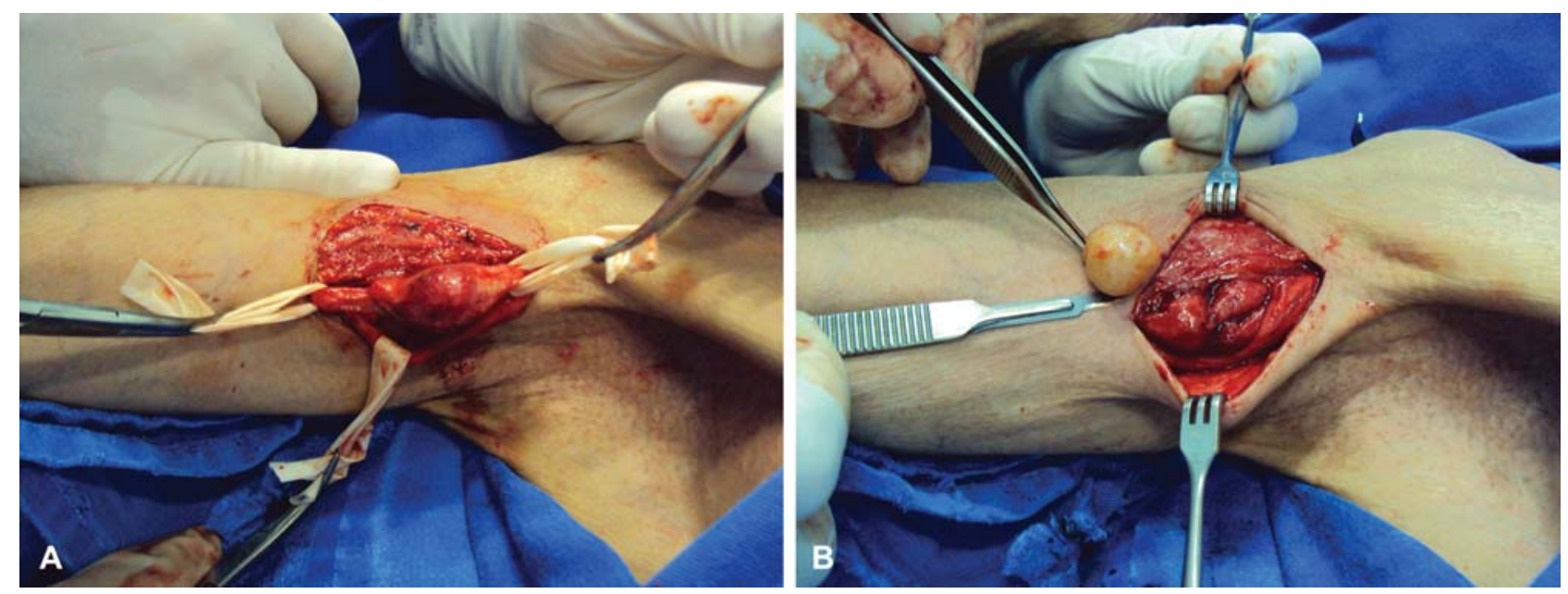

Fig. 4 Fotografias perioperatórias mostrando a disseção do tumor em região axilar direita (paciente número 7). (A) Evidencia-se isolamento do nervo com drenos de Penrose, facilitando exposição do tumor e permitindo que a peça tumoral seja dissecada de forma minuciosa, na tentativa de evitar complicações. (B) Área cruenta do leito do tumor e, ao lado, peça tumoral de aspecto amarelo-acinzentado, característico dos schwannomas.

prontuários, foram colhidas informações dos pacientes sobre idade, localização do tumor, região, lado, face e nervo acometidos, sinais e sintomas pré-operatórios - como dor, mobilidade, sinal de Tinel, alteração de sensibilidade e fraqueza -, data da cirurgia, complicações pós-operatórias, descrição do exame anatomopatológico, e presença ou não de recidivas.

O estudo foi submetido à avaliação e aprovação do Comitê de Ética em Pesquisa de nossa instituição.

\section{Resultados}

Com relação ao sexo, 10 pacientes eram do sexo feminino, e 10, do masculino. A média de idade foi de 50,85 anos, variando de 12 a 77 anos. No tocante à raça, todos os pacientes eram brancos.

Na - Tabela 1, encontram-se os dados referentes ao número de ordem dos pacientes, ao sexo, à idade e à data da cirurgia.
Na - Tabela 2, encontram-se os dados referentes ao número de ordem dos pacientes, aos sinais e sintomas préoperatórios (dor, mobilidade, sinal de Tinel, alteração da sensibilidade e fraqueza) e às complicações pós-cirúrgicas.

O membro superior foi acometido em 14 casos (70\%), e o membro inferior, em 6 (30\%). Quanto à região anatômica predominantemente ocupada pelo tumor, verificou-se comprometimento da face volar em 12 ocasiões (60\%), e da dorsal, em 8 (40\%).

Com relação à localização do tumor, seis estavam localizados na mão, quatro, no braço, três, no antebraço, um, na axila, três, na coxa, um, na perna, um, no tornozelo, e um, no pé. $O$ lado esquerdo foi acometido em 11 casos, enquanto o direito, em 9. 0 nervo ulnar foi o mais acometido, representando sete casos, seguido dos nervos radial e mediano.

Foi calculada a média aritmética das três dimensões do tumor com base na macroscopia. Obteve-se um valor de $3,31 \mathrm{~cm} \times 2,12 \mathrm{~cm} \times 1,68 \mathrm{~cm}$. 
Tabela 1 Número do paciente, sexo, idade e data da cirurgia dos pacientes da amostra

\begin{tabular}{|c|c|c|c|}
\hline Número de ordem & Sexo & Idade & Data da cirurgia \\
\hline 1 & $\mathrm{M}$ & 77 & fevereiro-02 \\
\hline 2 & $\mathrm{~F}$ & 32 & janeiro-06 \\
\hline 3 & $\mathrm{~F}$ & 53 & agosto-07 \\
\hline 4 & $\mathrm{~F}$ & 56 & fevereiro-10 \\
\hline 5 & $M$ & 12 & agosto-10 \\
\hline 6 & $\mathrm{~F}$ & 32 & agosto-10 \\
\hline 7 & $\mathrm{M}$ & 71 & dezembro-11 \\
\hline 8 & $\mathrm{M}$ & 75 & julho-12 \\
\hline 9 & $\mathrm{~F}$ & 66 & setembro-12 \\
\hline 10 & $\mathrm{~F}$ & 64 & novembro-12 \\
\hline 11 & $\mathrm{M}$ & 34 & dezembro-12 \\
\hline 12 & $\mathrm{M}$ & 53 & fevereiro-13 \\
\hline 13 & $\mathrm{M}$ & 24 & março-13 \\
\hline 14 & $\mathrm{~F}$ & 63 & abril-14 \\
\hline 15 & $\mathrm{~F}$ & 41 & outubro-14 \\
\hline 16 & $\mathrm{~F}$ & 77 & janeiro-15 \\
\hline 17 & $M$ & 37 & junho-16 \\
\hline 18 & $\mathrm{M}$ & 48 & junho-16 \\
\hline 19 & $\mathrm{~F}$ & 53 & abril-18 \\
\hline 20 & $\mathrm{M}$ & 49 & junho-18 \\
\hline
\end{tabular}

Na - Tabela 3, encontram-se dados referentes ao número de ordem dos pacientes, à localização, à face, e ao lado e nervo acometidos pelo tumor, além das suas dimensões.

No que se refere às complicações pós-operatórias, dois pacientes apresentaram parestesia, dois, hipoestesia, um, hiperestesia, e um, hipoestesia associada a parestesia. Não foi observado déficit motor em nenhum paciente no pós-operatório. No total, 6 (30\%) pacientes apresentaram alguma complicação pós-cirúrgica. No entanto, todas estas complicações foram transitórias, sendo que, após seis meses, todos os pacientes já não apresentavam mais nenhuma alteração de sensibilidade.

Não houve relatos de perda funcional ou sensitiva da região envolvida pelo nervo previamente acometido durante as reavaliações pós-operatórias tardias.

Até o momento, não foi identificado nenhum caso de recidiva tumoral.

\section{Discussão}

Embora raro, o schwannoma é o tumor benigno mais comum da bainha dos nervos periféricos. Acomete mais comumente a cabeça e o pescoço, seguido do tronco, e, depois, os membros superiores e inferiores. ${ }^{8,15,21}$

Há preferência para a face flexora dos membros, e ocorre tipicamente em indivíduos entre a quarta e sexta décadas de vida, sem predileção para sexo ou raça. Os nervos mais acometidos são o ulnar e o mediano, no membro superior, e o fibular e o tibial posterior, no inferior. ${ }^{8-12,16}$
A tríade clássica do schwannoma compreende a presença de massa, acompanhada de sinal de Tinel positivo e mobilidade no eixo transversal do membro. O quadro clínico pode ainda incluir dor, parestesia, hipoestesia e déficit motor. ${ }^{4,7,16}$

Rabari et al. ${ }^{22}$ realizaram estudo em que comentam sobre o atraso no diagnóstico do schwannoma. Jonathan et al. ${ }^{4}$ explicam que a ausência de um teste clínico específico, a variedade de sinais e sintomas, e a raridade da lesão são possíveis fatores que contribuem para esta maior dificuldade e atraso no diagnóstico. Além disso, trata-se de uma lesão que usualmente tem crescimento lento e é, em geral, indolor por vários anos, levando a uma demora na procura por atendimento. ${ }^{2,20}$

No Serviço de Anatomopatologia de nossa instituição, foram encontrados 20 casos de schwannomas, sendo que, destes, 14 foram correspondentes ao membro superior (70\%), e 6, ao membro inferior (30\%) (-Figura 5).

Em nosso estudo, houve predomínio dos membros superiores em relação aos inferiores, e da face flexora em relação à extensora. Os sexos feminino e masculino foram igualmente acometidos. A média de idade foi de 50,85, variando de 12 a 77 anos, com predileção para o intervalo entre a $4^{\mathrm{a}}$ e $6^{\mathrm{a}}$ décadas de vida. O nervo ulnar foi o mais acometido, seguido do nervo radial e mediano. Esses dados estão em consonância com os da literatura.

Com relação à amostra, todos os pacientes tinham massa palpável, 17 tinham dor à palpação, 17 tinham mobilidade no eixo transversal do membro, 6 apresentavam sinal de Tinel positivo, 6 tinham alteração de sensibilidade, e 4 apresentavam fraqueza.

Para auxiliar na elucidação diagnóstica do tumor, podem ser solicitadas a ultrassonografia e a ressonância magnética. À ressonância magnética, o tumor revela-se uma massa bem definida, usualmente fusiforme, em íntima relação com o nervo, isointenso em T1 e hiperintenso em T2, e pode apresentar o sinal do alvo. ${ }^{1,19}$ A ultrassonografia evidencia a estrutura fascicular dos nervos. Isto permite ao cirurgião definir o sítio, o tamanho e a relação com as estruturas adjacentes, o que auxilia na identificação da origem do tumor e do nervo acometido, de forma a contribuir no planejamento cirúrgico (escolha de material, equipamentos e métodos de aumento para microcirurgia), como salientado na seção Materiais e Métodos. ${ }^{2}$ A ressonância magnética, embora seja o exame de escolha, apresenta como desvantagem seu alto custo. Já o exame ultrassonográfico, apesar de ser mais barato, é operador-dependente. No entanto, o diagnóstico definitivo só é feito por meio do exame anatomopatológico. ${ }^{1,15,16}$ Os autores ressaltam que, por ser um tumor raro localizado em uma estrutura nobre, e pelo fato de um erro técnico-cirúrgico poder levar a grave déficit funcional, é muito importante que o profissional tenha em mente esta hipótese diagnóstica para evitar mau planejamento cirúrgico.

O tratamento preconizado é a intervenção cirúrgica precoce, que, apesar de ter um prognóstico favorável, comumente apresenta complicações pós-cirúrgicas temporárias, como distúrbios sensitivos e/ou motores. ${ }^{6,7}$ Portanto, é de extrema importância que os pacientes sejam informados quanto aos riscos a que estarão expostos durante e após o procedimento cirúrgico. ${ }^{4}$ Em nosso estudo, só houve complicações sensitivas, 
63420 pacientes acometidos por schwannoma nos membros superior e inferior Galbiatti et al.

Tabela 2 Número do paciente, sintomas e sinais pré-operatórios (dor, mobilidade, sinal de Tinel, alteração da sensibilidade, fraqueza) e complicações pós-cirúrgicas

\begin{tabular}{|c|c|c|c|c|c|c|}
\hline Número do paciente & Dor & Mobilidade & Sinal de Tinel & $\begin{array}{l}\text { Alteração da } \\
\text { sensibilidade }\end{array}$ & Fraqueza & Complicação pós-cirúrgica \\
\hline 1 & + & + & + & - & + & Hipoestesia \\
\hline 2 & + & + & - & - & + & - \\
\hline 3 & + & + & - & - & - & - \\
\hline 4 & - & + & - & - & - & Parestesia \\
\hline 5 & + & + & - & - & - & - \\
\hline 6 & + & - & - & + & - & - \\
\hline 7 & + & + & - & - & - & - \\
\hline 8 & + & + & - & + & - & Hipoestesia \\
\hline 9 & + & + & + & - & + & - \\
\hline 10 & + & - & + & + & - & - \\
\hline 11 & + & + & - & - & - & - \\
\hline 12 & + & + & - & - & - & - \\
\hline 13 & + & + & - & + & - & - \\
\hline 14 & + & + & - & - & - & - \\
\hline 15 & + & + & + & + & + & Parestesia e hipoestesia \\
\hline 16 & + & + & - & - & - & Hiperestesia \\
\hline 17 & + & - & + & - & - & Parestesia \\
\hline 18 & - & + & - & - & - & - \\
\hline 19 & - & + & - & - & - & - \\
\hline 20 & + & + & + & + & - & - \\
\hline
\end{tabular}

Tabela 3 Número do paciente, localização, face, lado e nervo acometidos, bem como as dimensões dos tumores

\begin{tabular}{|c|c|c|c|c|c|}
\hline Número do paciente & Localização & Face & Lado & Nervo & Dimensões (em cm) \\
\hline 1 & Mão & Volar & Esquerdo & Ulnar & $1,7 \times 1,2 \times 0,9$ \\
\hline 2 & Antebraço & Dorsal & Esquerdo & Interósseo posterior & $1,4 \times 0,9 \times 0,9$ \\
\hline 3 & Pé & Dorsal & Direito & Ramo cutâneo dorsal lateral do nervo sural & $3,6 \times 1,9 \times 1,5$ \\
\hline 4 & Mão & Dorsal & Direito & Ulnar & $3,5 \times 2,7 \times 2,0$ \\
\hline 5 & Braço & Volar & Direito & Nervo cutâneo medial do braço & $7,5 \times 3,4 \times 3,5$ \\
\hline 6 & Tornozelo & Dorsal & Esquerdo & Tibial posterior & $2,3 \times 1,8 \times 1,2$ \\
\hline 7 & Axila & Volar & Direito & Músculo-cutâneo & $1,5 \times 1,5 \times 1,5$ \\
\hline 8 & Braço & Dorsal & Direito & Radial & $10,5 \times 6,5 \times 4,0$ \\
\hline 9 & Braço & Dorsal & Direito & Radial & $2,0 \times 1,5 \times 1,0$ \\
\hline 10 & Mão & Volar & Esquerdo & Mediano & $0,9 \times 0,7 \times 0,7$ \\
\hline 11 & Coxa & Dorsal & Direito & Cutâneo femoral lateral & $3,6 \times 2,2 \times 1,8$ \\
\hline 12 & Perna & Volar & Esquerdo & Fibular & $4,3 \times 2,5 \times 1,6$ \\
\hline 13 & Antebraço & Volar & Esquerdo & Ulnar & $1,8 \times 1,8 \times 1,4$ \\
\hline 14 & Coxa & Dorsal & Esquerdo & Safeno & $4,8 \times 3,0 \times 2,7$ \\
\hline 15 & Braço & Volar & Esquerdo & Ulnar & $2,5 \times 1,8 \times 1,6$ \\
\hline 16 & Coxa & Volar & Esquerdo & Femoral & $7,5 \times 5,0 \times 4,2$ \\
\hline 17 & Mão & Volar & Direito & Mediano & $1,6 \times 1,1 \times 0,5$ \\
\hline 18 & Mão & Volar & Direito & Ramo digital palmar próprio do nervo ulnar & $1,3 \times 0,4 \times 0,6$ \\
\hline 19 & Mão & Volar & Esquerdo & Ulnar & $2,5 \times 1,3 \times 1,0$ \\
\hline 20 & Antebraço & Volar & Esquerdo & Ulnar & $1,5 \times 1,2 \times 1,0$ \\
\hline
\end{tabular}


Membro superior

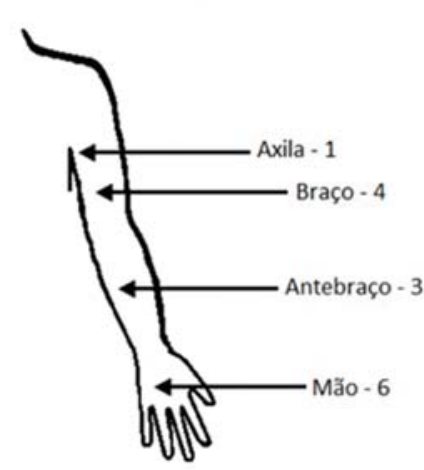

\section{Membro inferior}

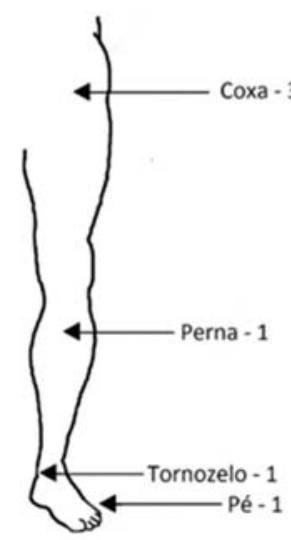

Fig. 5 Ilustração de membro superior e inferior evidenciando a distribuição topográfica dos tumores encontrados no estudo.

compreendendo 30\% da amostra. Todas essas complicações foram transitórias.

Gosk et al. ${ }^{10}$ e Ujigo et al. ${ }^{23}$ comentam em seus estudos sobre fatores que podem afetar a evolução do tratamento e que podem acarretar maior risco de complicações pós-operatórias. Tais fatores são: sinal de Tinel positivo, tamanho e localização do tumor, característica histológica, severidade dos sintomas manifestados antes da operação, tumor de história mais longa, e técnica cirúrgica inadequada.

Consideramos que, entre esses fatores, a presença do sinal de Tinel deve ser destacada, visto que já demonstra uma alteração neurológica local. Em nossa casuística, 50\% dos pacientes com sinal de Tinel positivo apresentaram complicações pós-operatórias, enquanto que, nos pacientes que não o apresentaram, a taxa de complicações foi de $21,4 \%$. Tais dados confirmam a valorização da presença do sinal de Tinel no prognóstico.

A recorrência e a malignização do schwannoma são raras. ${ }^{6,8,15,16}$ Em nosso estudo, não houve nenhum caso de recidiva. Com relação à malignização, encontramos um caso, no paciente número 8 , no qual foi encontrado um schwannoma maligno de grau nuclear e índice mitótico moderados, com áreas epitelioides, invasivo do subcutâneo e do tecido fibromuscular até o periósteo, com bordas tumorais de padrão expansivo, e presença de áreas focais de inflamação estromal, necrose e hemorragia.

Por se tratar de um Serviço de Ortopedia, optou-se por avaliar os pacientes acometidos por schwannoma apenas nos membros superiores e inferiores. Além disso, há poucos estudos com série de casos em nosso país sobre esse tema. Por esses motivos, os autores decidiram realizar uma análise do perfil epidemiológico, bem como da evolução natural e intervenção nesta enfermidade em nosso serviço, destacando que os exames complementares devem auxiliar na formulação da hipótese de schwannoma, que exigirá material e técnica microcirúrgica compatíveis com a nobreza da estrutura acometida. Diante dos resultados, pode-se considerar que os dados obtidos estão em concordância com os descritos na literatura.

\section{Conclusão}

O schwannoma é uma lesão rara e de difícil diagnóstico. Deve sempre ser considerada como hipótese quando se estiver diante de um tumor de partes moles acometendo os membros, especialmente quando associado à presença de mobilidade no eixo transversal e sintomas neurológicos, dentre os quais, o sinal de Tinel deve ser valorizado, visto sua maior correlação com as complicações pós-operatórias. Os pacientes devem ser informados quanto às possíveis complicações, que são frequentes (cerca de 30\%), mas, geralmente, transitórias.

Conflito de Interesses

Os autores declaram não haver conflito de interesses.

\section{Referências}

1 Gosk J, Gutkowska O, Urban M, Wnukiewicz W, Reichert P, Ziółkowski P. Results of surgical treatment of schwannomas arising from extremities. BioMed Res Int 2015;2015:547926

2 Kim SM, Seo SW, Lee JY, Sung KS. Surgical outcome of schwannomas arising from major peripheral nerves in the lower limb. Int Orthop 2012;36(08):1721-1725

3 Knight DMA, Birch R, Pringle J. Benign solitary schwannomas: a review of 234 cases. J Bone Joint Surg Br 2007;89(03):382-387

4 Jonathan CK, Fu-Keung IP, Tak-Chuen W, et al. Neurilemmomas of the hand: a review of the clinical presentation, surgical outcome, and potential risk factors. J Orthop 2017;22(01):48-51

5 Kransdorf MJ. Benign soft-tissue tumors in a large referral population: distribution of specific diagnoses by age, sex, and location. AJR Am J Roentgenol 1995;164(02):395-402

6 Ozdemir O, Ozsoy MH, Kurt C, Coskunol E, Calli I. Schwannomas of the hand and wrist: long-term results and review of the literature. J Orthop Surg (Hong Kong) 2005;13(03):267-272

7 Park MJ, Seo KN, Kang HJ. Neurological deficit after surgical enucleation of schwannomas of the upper limb. J Bone Joint Surg Br 2009;91(11):1482-1486

8 Rammohan R, Gupta P, Maini L, Gautam VK. Neurilemmoma of median nerve. J Clin Orthop Trauma 2014;5(01):33-37

9 Tang CYK, Fung B, Fok M, Zhu J. Schwannoma in the upper limbs. BioMed Res Int 2013;2013:167196

10 Gosk J, Gutkowska O, Mazurek P, Koszewicz M, Ziółkowski P. Peripheral nerve tumours: 30 -year experience in the surgical treatment. Neurosurg Rev 2015;38(03):511-520

11 Ahmed AR, Watanabe H, Aoki J, Shinozaki T, Takagishi K. Schwannoma of the extremities: the role of PET in preoperative planning. Eur J Nucl Med 2001;28(10):1541-1551

12 Omezzine SJ, Zaara B, Ben Ali M, Abid F, Sassi N, Hamza HA. A rare cause of non discal sciatica: schwannoma of the sciatic nerve. Orthop Traumatol Surg Res 2009;95(07):543-546

13 Sandberg K, Nilsson J, Søe Nielsen N, Dahlin LB. Tumours of peripheral nerves in the upper extremity: a 22-year epidemiological study. Scand J Plast Reconstr Surg Hand Surg 2009;43(01): 43-49

14 Forthman CL, Blazar PE. Nerve tumors of the hand and upper extremity. Hand Clin 2004;20(03):233-242, v

15 Sá A, Azevedo LN, Cunha L. Schwannoma do membro superior: análise retrospectiva de 17 casos. Acta Med Port 2016;29(09): 519-524

16 Adani R, Baccarani A, Guidi E, Tarallo L. Schwannomas of the upper extremity: diagnosis and treatment. Chir Organi Mov 2008; 92(02):85-88

17 Levi AD, Ross AL, Cuartas E, Qadir R, Temple HT. The surgical management of symptomatic peripheral nerve sheath tumors. Neurosurgery 2010;66(04):833-840 
63620 pacientes acometidos por schwannoma nos membros superior e inferior Galbiatti et al.

18 Sawada T, Sano M, Ogihara H, Omura T, Miura K, Nagano A. The relationship between pre-operative symptoms, operative findings and postoperative complications in schwannomas. J Hand Surg [Br] 2006;31(06):629-634

19 Adani R, Tarallo L, Mugnai R, Colopi S. Schwannomas of the upper extremity: analysis of 34 cases. Acta Neurochir (Wien) 2014;156 (12):2325-2330

20 Hernández DG, González RM, Mesa JM, et al. Schwannoma of the median nerve at the wrist: a case report and review of literature. Ortho Rheum Open Access J 2017;4(05):555-648
21 Mizushima H. Neurological deficits before and after surgical resection of Schwannomas in the upper extremities. J Reconstr Microsurg 2016;32(05):371-377

22 Rabari Y, Prasad DV, Thadeshwar K, et al. Schwannomas - the problem in delay diagnosis. Int J Orthop Sci 2017;3(01): 459-462

23 Ujigo S, Shimose S, Kubo T, Fujimori J, Ochi M. Therapeutic effect and risk factors for complications of excision in 76 patients with schwannoma. J Orthop Sci 2014;19(01):150-155 